\title{
Article
}

\section{Comparison of Treatment Rates of Depression After Stroke Versus Myocardial Infarction: A Systematic Review and Meta-Analysis of Observational Data}

Ladwig, Simon, Zhou, Zien, Xu, Ying, Wang, Xia, Chow, Clara K., Werheid, Katja and Hackett, Maree

Available at http://clok.uclan.ac.uk/24740/

Ladwig, Simon, Zhou, Zien, Xu, Ying, Wang, Xia, Chow, Clara K., Werheid, Katja and Hackett, Maree ORCID: 0000-0003-1211-9087 (2018) Comparison of Treatment Rates of Depression After Stroke Versus Myocardial Infarction: A Systematic Review and Meta-Analysis of Observational Data. Psychosomatic Medicine, 80 (8). pp. 754-763. ISSN 0033-3174

It is advisable to refer to the publisher's version if you intend to cite from the work. http://dx.doi.org/10.1097/PSY.0000000000000632

For more information about UCLan's research in this area go to http://www.uclan.ac.uk/researchgroups/ and search for <name of research Group>.

For information about Research generally at UCLan please go to http://www.uclan.ac.uk/research/

All outputs in CLoK are protected by Intellectual Property Rights law, including Copyright law. Copyright, IPR and Moral Rights for the works on this site are retained by the individual authors and/or other copyright owners. Terms and conditions for use of this material are defined in the policies page. 
Title: Comparison of treatment rates in depression after stroke versus myocardial infarction. A systematic review and meta-analysis of observational data. Authors: Simon Ladwig (MSc) $)^{1,2}$, Zien Zhou (MD) ${ }^{3,4}$, Ying Xu (MD) ${ }^{4,5}$, Xia Wang (PhD) ${ }^{4}$, Clara K. Chow $(\mathrm{PhD})^{6,7,8}$, Katja Werheid $(\mathrm{PhD})^{2,9}$, \& Maree L. Hackett $(\mathrm{PhD})^{4,10,11}$

1. Department of Psychology, University of Potsdam, Potsdam, Germany

2. Department of Neurology, Ernst von Bergmann Hospital, Potsdam, Germany

3. Department of Radiology, Ren Ji Hospital, School of Medicine, Shanghai Jiao Tong University, Shanghai, China

4. Mental Health, The George Institute for Global Health, The University of New South Wales, Sydney, Australia

5. Sydney School of Public Health, Faculty of Medicine and Health, The University of Sydney, Sydney, Australia

6. Westmead Applied Research Centre, Faculty of Medicine and Health, University of Sydney, Sydney, Australia

7. Department of Cardiology, Westmead Hospital, Sydney, Australia

8. Cardiovascular Division, The George Institute for Global Health, The University of New South Wales, Sydney, Australia

9. Department of Psychology, Humboldt University, Berlin, Germany

10. Sydney Medical School, The University of Sydney, Sydney, New South Wales, Australia

11. The Faculty of Health and Wellbeing, The University of Central Lancashire, Preston, United Kingdom

Word count: 5.932

Tables: 2

Figures: 2

Corresponding author details:

Simon Ladwig

Klinikum Ernst von Bergmann

Klinik fuer Neurologie

Charlottenstrasse 72

14467 Potsdam

Email: simon.ladwig@uni-potsdam.de

Phone: 0049 331. 241 - 37164

Fax: 0049331.241 - 37100 
Treatment rates in PSD and PMID

1

2

3

4 University (2016-2018) and a Scientia PhD Scholarship from the UNSW Sydney (2018-2022).

5 Clara Chow was supported by National Health and Medical Research Council Career Development

6 Fellowship (Level 2) (APP1105447) and co-funded by a Heart Foundation Future Leader

7 Fellowship. Maree Hackett was in receipt of a National Heart Foundation (Australia) Future Leader 
Treatment rates in PSD and PMID

2 Objective: Depression after stroke and myocardial infarction are common but often assumed to be undertreated without reliable evidence being available. Thus, we aimed to determine treatment rates and investigate the application of guidelines in these conditions.

5 Methods: Databases MEDLINE, EMBASE, PsycInfo, Web of Science, CINAHL, and Scopus were 6 systematically searched without language restriction from inception to $06 / 30 / 2017$. Prospective observational studies with consecutive recruitment reporting any antidepressant treatment in adults with depression after stroke or myocardial infarction were included. Random effects models were used to calculate pooled estimates of treatment rates.

10 Results: 55 studies reported 32 stroke cohorts $(n=8,938$; pooled frequency of depression $=34 \%$,

$1195 \%$ CI 29 to $38 \%)$ and 17 myocardial infarction cohorts $(n=10,767$; pooled frequency of

12 depression $=24 \%, 95 \%$ CI 20 to $28 \%$ ). In 29 stroke cohorts, $24 \%$ (95\% CI 20 to $27 \%$ ) of 2,280 depressed people used antidepressant medication. In 15 myocardial infarction cohorts, $14 \%$ (95\% CI 8 to $19 \%$ ) of 2,381 depressed people used antidepressant medication indicating a lower treatment rate than in stroke. After stroke, treatment with antidepressant medication was more frequent in moderate to severe $(22 \%, 95 \%$ CI 14 to $29 \%)$ than in mild depression $(9 \%, 95 \%$ CI 7 to $12 \%)$. Two studies reported use of psychosocial interventions, indicating that $<10 \%$ of participants were treated.

Conclusions: Despite the high frequency of depression after stroke and myocardial infarction and the existence of efficacious treatment strategies, people often remain untreated. Strategies to increase the use of efficacious treatments are needed. 
Treatment rates in PSD and PMID

2

$3 \mathrm{ADT}(\mathrm{s})$ - Antidepressant drug(s)

4 BDI(-II) - Beck Depression Inventory(-II)

5 CI - Confidence interval

6 DSM - Diagnostic and Statistical Manual of Mental Disorders

7 ICD - International Classification of Diseases

$8 \mathrm{MeSH}$ - Medical Subject Headings

9 MI - Myocardial infarction

10 OECD - Organisation for Economic Co-operation and Development

11 PMID - Post-myocardial infarction depression

12 PSD - Post-stroke depression

13 SSRIs - Selective Serotonin Reuptake Inhibitors

14 WHO - World Health Organization

15

16

17 
Treatment rates in PSD and PMID

Introduction

Stroke and myocardial infarction (MI) are among the most common causes of disability worldwide and their burden is likely to increase $(1,2)$. Both diseases share a sudden onset, a threat to life (3), a need for long term rehabilitation $(4,5)$, and similar lifestyle risk factors (6). Additionally, depression affects $31 \%$ of all people at any time up to five years after stroke (7) and $28 \%$ of all people within two years of MI (8). These conditions, referred to as post-stroke depression (PSD) and post-MI depression (PMID), have an adverse impact on rehabilitation, including impaired functional outcome, reduced quality of life, lower medication adherence, increased risk of recurrent events, and higher mortality (8-10). The efficacy of antidepressant drugs (ADTs), but not talking therapies, has been shown to be effective for the treatment of PSD, albeit with an associated increase in adverse events (11). People with PMID have been shown to benefit from ADTs and psychosocial interventions ${ }_{2}$ including relaxation therapy $(12,13)$. Furthermore, ADTs are recommended in moderate to severe PSD and PMID while those with milder symptoms should be closely monitored $(14,15)$. Individual studies have also evaluated electroconvulsive therapy $(11,16)$, herbal medicine (14), and non-invasive brain stimulation (17), but their efficacy has not been comprehensively demonstrated.

Proof of efficacy enhances the public's beliefs about an intervention and thereby leads to better implementation (18). Nevertheless, use of ADTs is not a reliable indicator of adequate management of PSD. ADTs were found to reduce dependency, disability, neurological impairment, and pain after stroke which may be attributable to beneficial effects on drive and motivation as well as on central nervous functioning $(19,20)$. After MI,-while-ADTs did not improvecould not be shown to improve cardiac prognosis (21-23) which may be due to low power for detecting mortality reduction in these trials (24). However, based on the current evidence people with depression after stroke may have some indications for the prescription of ADTs, which do not apply to MI. 
Treatment rates in PSD and PMID

\section{Methods}

This systematic review was undertaken according to the MOOSE guidelines for meta-analyses of observational studies (31) and reported according to the PRISMA statement (32). The protocol was prospectively registered in PROSPERO (CRD42016051232).

\section{Study selection}

We included prospective observational studies with consecutive recruitment reporting data on treatment use at any given time-point after stroke or MI. Randomized controlled trials, case-control studies, and cross-sectional studies were excluded. Cohorts were included if participants (1) were $\geq$ 18 years, (2) had a clinical diagnosis of stroke or MI, and (3) were assessed for depressive symptoms using defined scores on standard screening instruments or depressive disorders (minor depression, dysthymia, major depression) applying ICD or DSM criteria. Finally, studies were included if they (4) reported the frequency of use of ADTs, psychosocial interventions, herbal medicine, electroconvulsive therapy or non-invasive brain stimulation for the treatment of depression. Psychosocial interventions were defined as any treatment including telemedical or direct patientprofessional interaction ranging from counselling to psychotherapy. Interventions with the sole purpose of education, information or social transfer, and occupational therapy were excluded. 
Treatment rates in PSD and PMID

Data sources and extraction

The literature search was conducted on MEDLINE, EMBASE, PsycInfo, Web of Science, CINAHL, and Scopus from inception to 06/30/2017. Databases were searched using MeSH terms and related keywords for stroke OR myocardial infarction AND depression AND prospective study design. The search strategy for MEDLINE is accessible at http://www.crd.york.ac.uk/PROSPEROFILES/ 51232_STRATEGY_20161010.pdf and was adjusted for other databases. After searching and excluding irrelevant studies via title and abstract, eligibility was examined using full text articles. Reference lists of included articles and related review articles were manually searched. In an attempt to access all published studies worldwide, 17 non-English articles were reviewed in full text and translated from Chinese, Czech, Danish, French, German, Portuguese, Russian, and Spanish. Study quality was assessed by grouping studies into three categories representing completeness of case-selection (7). The first group of population-based studies, considered the highest (least biased) quality, consisted of studies that attempted to recruit all people with stroke or MI, including those not admitted to hospital for acute care. The other two categories were hospital-based studies, which included all inpatients recruited from acute care medical wards in general hospitals, and rehabilitation-based studies, which included patients from rehabilitation wards or stroke/cardiac care units. Treatment rates were also pooled and compared among WHO world regions (33) and between OECD-member and non-member countries, if applicable. Furthermore, treatment rates were pooled and compared among decades of publication (before 2000, 2000-2009, since 2010).

Study reports with evidence of overlapping recruitment sites, study dates, grant funding numbers, and similar or identical reported patient characteristics were considered to be from the same cohort. If several articles reported data from the same cohort, data were taken from the first publication of a given time-point. If multiple instruments were used to assess depressive symptoms and their treatment at the same time point, data showing the highest proportion treated were included. 
Treatment rates in PSD and PMID

1 All authors were contacted for missing or additional data and to confirm suspected overlapping cohorts. Additional data were included if received before 30/09/2017.

4 Extracted data were stratified according to case selection and time of assessment after stroke or MI. Data assessed up to three months after the ictus were categorized as short-term, from three up to twelve months as medium-term and twelve months or later as long-term. Depression was categorised as mild (minor, mild depression; dysthymia) or moderate/severe (moderate, severe, major depression) according to the categories applied by study authors.

9 Frequencies of depressive symptoms and treatment use at the first assessment were pooled using the random effects model of DerSimonian and Laird (34). Sensitivity analyses included the comparison with fixed effect models, treatment use at last assessment, cohort size: small $(n<100)$ and large $(n>$ 100), and interviewer-administered vs. self-completed questionnaires to screen for depression. Publication bias was assessed by inspecting funnel plots and conducting Egger's regression (35). Subgroup meta-analyses included patient groups with mild, moderate/severe, and no depression receiving treatment.

The applied search strategies identified over 46,000 articles, of which 625 were reviewed in full-text. 32 stroke and 17 MI studies (in 55 manuscripts) were included. Authors of 20 studies provided additional unpublished data (36-55). The review process is illustrated in Figure 1. 
Treatment rates in PSD and PMID

1 Description of the study samples Population deseription

Commented [LS4]: J.C3

2 People with stroke were assessed between two weeks and five years after the ictus. The minimum

3 age criterion of $\geq 18$ years could not be confirmed in seven studies $(56,66,67,71,74-76)$. One study

4 (59) included people $\geq 15$ years of age and was included because of an assumed small number of

5 people under 18 years and a large sample size. Further study details are listed in Table 1. The pooled

6 frequency of PSD in 32 cohorts was 34\% (95\% confidence interval [CI] 29 to 38\%) with significant

7 heterogeneity among studies $(p<.001)$ and a significant intercept in Egger's regression $(p<.001)$

8 indicating that smaller studies reported higher frequencies.

9

INSERT [TABLE 1] ABOUT HERE

People with MI were assessed between one day and 18 months after the ictus. The age criterion could not be confirmed in three studies $(79,82,84)$. Further study details are listed in Table 2 . The pooled frequency of PMID in 17 cohorts was 24\% (95\% CI 20 to 28\%) with significant heterogeneity $(p<.001)$ and a non-significant intercept in Egger's regression $(p=.19)$.

\section{INSERT [TABLE 2] ABOUT HERE-}

\section{Treatment of PSD}

No treatment other than ADTs or psychosocial interventions were reported. One stroke study reported use of psychotherapy (none of the 89 people with PSD received psychotherapy) (60), and another reported if people were referred to a psychiatric service in addition to receiving ADTs (none of 11 people with PSD were referred) (41). The studies did not specify these treatments further. Hence, 31 studies reported use of ADTs. One of these did not report the cut-off used for depression 
Treatment rates in PSD and PMID $22>.30)$

23

5 . 17

assessment and was excluded from analyses (55). Visual investigation of the funnel plot led to exclusion of a further study representing an outlier with small sample (62). Results of sensitivity analyses were identical if this study was in- or excluded.

Finally, 29 cohorts consisting of 8,634 people with stroke were included in random effects analyses of ADT use. Frequency of PSD in these cohorts (32\%, 95\% CI 27 to 37\%) was not significantly different from the frequency in all 32 cohorts. The pooled estimate of ADT use was $24 \%$ (95\% CI 20 to $27 \%)$ in 2,280 people with PSD with significant heterogeneity among studies $(p<.001)$ and a non-significant intercept in Egger's regression $(p=.082)$. Sensitivity analyses did not produce any significantly different result. The forest plot (Fig. 2) illustrates the pooled estimates for different time windows and recruitment types. There was no significant difference in treatment rates among population-based $(21 \%, 95 \%$ CI 14 to $28 \%)$, hospital-based (21\%, 95\% CI 15 to $27 \%)$, and rehabilitation-based studies (28\%, 95\% CI 22 to $34 \%)$. Analyses comparing pooled treatment rates between cohorts from different WHO world regions (33), between OECD member and non-member countries as well as among decades of publication produced no significantly different results.

Data on ADT use could be extracted from three cohorts with mild depression $(41,42,71)$ and six cohorts with moderate to severe depression $(37,39,41,42,67,71)$. ADTs were significantly less often used in PSD with mild depression $(9 \%, 95 \%$ CI 7 to $12 \%)$ than in PSD with moderate to severe depression $(22 \%, 95 \%$ CI 14 to $29 \%)$. Heterogeneity of studies was not significant in either group ( $p$

Furthermore, twelve studies reported ADT use in non-depressed people with stroke $(39-41,54,57$, $58,64,69-71,75,77)$. The pooled estimate of these frequencies was $11 \%$ (95\% CI 7 to $14 \%$ ) with 
Treatment rates in PSD and PMID

significant heterogeneity among studies $(p<.001)$ and a non-significant intercept in Egger's regression $(p>90)$. Additionally, one study reported that $18 \%$ of those not depressed and treated at 12 months were previously depressed at 3 months (57) and one study reported that $64 \%$ who were not depressed and treated at 6 months were previously depressed at 7 weeks (54).

\section{Treatment of PMID}

No treatment other than ADTs or psychosocial interventions were reported. One study reported the use of psychopharmacology and/or psychotherapy by 3 of 5 with PMID (60.0\%) (78), one study reported use of psychiatric treatment by 3 of 18 with PMID (16.6\%) (82), and one study reported use of psychosocial interventions for depression by 72 of 759 with PMID (9.5\%) (81) without further description of these interventions.

The 15 studies reporting use of ADTs consisted of 10,635 with MI who were included in the random effects analyses. The pooled frequency of PMID in these cohorts (25\%, 95\% CI 21 to 30\%) was not significantly different from the frequency in all 17 cohorts. The pooled frequency of ADT use was $14 \%$ (95\% CI 8 to $19 \%$ ) in 2,381 with PMID and hence, significantly lower than in PSD. Heterogeneity among studies was significant $(p<.001)$ with a non-significant intercept in Egger's regression $(p=.062)$. The estimate did not change in sensitivity analyses. Furthermore, people in hospital-based studies received ADTs more often (19\%, 95\% CI 15 to $24 \%)$ than people in rehabilitation-based studies $(8 \%, 95 \%$ CI 4 to $13 \%)$. The ten short-term studies showed a pooled frequency of $16 \%$ (95\% CI 11 to $21 \%)(44,45,48-53,80,81)$, the three medium-term studies a frequency of $12 \%$ (95\% CI 0 to $25 \%$ ) $(46,83,84)$, and the two long-term studies a frequency of $6 \%$ (95\% CI 1 to $10 \%)(47,79)$ demonstrating a significant difference between frequencies in the shortand the two long-term rehabilitation-based studies. Comparing pooled treatment rates between cohorts from the European region and the region of the Americas as well as among decades of publication yielded no significant difference. 
Treatment rates in PSD and PMID

\section{Discussion}

This meta-analysis provides the first comprehensive evidence of depression treatment rates after stroke and myocardial infarction. Although more PSD cohorts could be identified, PMID cohorts were usually larger resulting in similar numbers of people being included. The frequencies of depressive symptoms were in the same range as in other systematic reviews on PSD (7) and PMID (8), supporting the generalisability of our findings. Only a few study authors reported the use of treatments other than ADTs $(41,60,78,81,82)$. This may be explained by the lack of evidence of other efficacious treatments for people with PSD (11), whereas pharmacological and psychological psychosocial interventions have proven efficacious for PMID-(12). This may also be due to the investigation of more innovative interventions in PMID like e.g. the telephone-based collaborative care program of the MOSAIC trial (85). - -As only few or no people used psychosocial interventions in the studies reporting these treatments and use of psychosocial interventions may be documented less often in outpatient settings, this finding may also be attributed to reporting and measurement biases.

Every fourth person with PSD and every seventh person with PMID reported using ADTs indicating that only a small proportion of people with depression after stroke and MI receives evidence-based treatment. Clinical trials have proven the efficacy of ADTs in both disorders $(11,12)$ but they are only effective if they are prescribed and taken (18). Additionally, some studies indicate higher mortality in people with untreated PSD and PMID $(81,86)$. However, the ENRICHD trial did not show a reduction of late mortality in people with PMID who were treated with cognitive behavioral therapy and sertraline but demonstrated increased late mortality in people whose depression is refractory to treatment (22). Possible reasons for undertreatment include insufficient assessment and follow-up of psychological status $(47,87)$ as well as uncertainty due to comorbidities and polypharmacy, which is common in people with stroke and MI (25). Furthermore, depression may 
Treatment rates in PSD and PMID

be perceived as a natural reaction to stroke or MI which therefore is assumed to require no further treatment (88). Depressive symptoms such as lack of energy, hopelessness and withdrawal may also contribute to people stopping rehabilitation, follow-up visits and medications (46). Additionally, patients may want to avoid the label and associated stigma of mental illness and therefore, withdraw from treatment (89). Frequency of treatment was not different based on case selection in PSD, indicating similar health care practices across all settings. Treatment rates in rehabilitation-based PMID cohorts were significantly lower than in hospital-based cohorts, which contrasts especially with stroke cohorts showing the descriptively highest frequency of treatment in rehabilitation settings. The difference between MI cohorts may be attributable to better access of people with MI to mental health professionals in hospital compared to rehabilitation due to counseleil service. Although guidelines list psychological evaluation as an essential part of cardiac rehabilitation (84), evidence suggests low psychological expertise in this setting (85). While cardiac rehabilitation usually focuses on the prevention of recurrent events using pharmacological and lifestyle interventions (84), stroke rehabilitation includes neuropsychological assessment and treatment of cognitive impairment (86). Therefore, mental health expertise may be higher in hospital and stroke rehabilitation compared to cardiac rehabilitation possibly resulting in better recognition and treatment of depressive symptoms.

Treatment rates in PSD and PMID did not differ between world regions (33) or by OECD membership where most cohorts were from Europe, North America and/or OECD countries. Additionally, treatment rates did not differ depending on year of publication despite major developments in the health care of people with stroke and MI over the last decades. Three studies $(37,40,69)$ distinguishing classes of ADTs reported SSRIs being most frequently used in PSD which is in line with research favoring SSRIs as a pharmacological treatment (19).

PSD was more frequently treated than PMID and a tenth of non-depressed people received ADTs after stroke. This may be based on the clinical consideration of findings supporting the efficacy of 
Treatment rates in PSD and PMID

1 ADTs for physical and functional rehabilitation after stroke $(19,20)$. Additionally, people with more

2 severe PSD received ADTs more frequently which is in keeping with evidence-based guideline

3 recommendations (15), better recognition (90), and higher need (91).

4 While our findings are limited by the high variability of the assessment tools used, criteria for

5 depressive symptoms, assessment times, methods for collecting data on treatment use, and the

6 selection criteria within the cohorts, they remain consistent in sensitivity analyses. As a further

7 strength of this meta-analysis, we included many unpublished data sets which were provided by

8 original authors. However, it must be noted that the reported use of antidepressants may not indicate

9 adequate treatment of depression as they may also be described to improve physical and functional

10 rehabilitation or treat anxiety $(19,20)$. Furthermore, this meta-analysis excluded interventions solely

Commented [LS8]: J.C4

11 providing information or education as they were not investigated in randomized controlled trials up

12 to now. Nevertheless, people with PSD or PMID may benefit from these interventions. Finally, many

Commented [LS9]: R2.C2

13 cohorts had to be excluded as they assessed depressive symptoms in cohorts of people with coronary

14

diseases generally, rather than solely MI. We are unclear if, or to what extent, this may bias our results.

While the specific determinants of treatment use in PSD and PMID may need further investigation, the considerable undertreatment found in our study indicates a need for screening for depression after stroke and MI $(8,9)$ and clear management protocols which include reassessment and stopping guidelines for use in healthcare settings. As guidelines already include similar recommendations ( 9 , $14,15)$, specific education of health professionals is essential to close the gap to clinical practice, increase treatment rates, and thereby reduce the significant burden of PSD and PMID globally (1). 
Treatment rates in PSD and PMID

2 The authors would like to thank all corresponding study authors for providing additional data and

3 information. We also thank Mansur Kutlubaev (Russian), Lorena Ataíde Lopes (Portuguese), and

4 Marek Čontošfalský (Czech) for translating non-English language articles.

5 
Treatment rates in PSD and PMID

2

$3 \quad$ Fig.1 PRISMA flow diagram of literature review process

$4 \quad$ Fig.2 Forest plot of antidepressant drug treatment in people with post-stroke depression

5

6

7

8

9

10

11

12

13

14

15

16

17

18

19

20

21

22 


\section{References}

1. Mathers CD, Loncar D. Projections of global mortality and burden of disease from 2002 to 2030. PLoS Med. 2006;3:e442.

2. Truelsen T, Piechowski - Jóźwiak B, Bonita R, Mathers C, Bogousslavsky J, Boysen G. Stroke incidence and prevalence in Europe: a review of available data. Eur J Neurol. 2006;13:581-98.

3. Lozano R, Naghavi M, Foreman K, Lim S, Shibuya K, Aboyans V, Abraham J, Adair T, Aggarwal $\mathrm{R}$, Ahn SY, al e. Global and regional mortality from 235 causes of death for 20 age groups in 1990 and 2010: A systematic analysis for the Global Burden of Disease Study 2010. Lancet. 2013;380:2095-128.

4. Duncan PW, Zorowitz R, Bates B, Choi JY, Glasberg JJ, Graham GD, Katz RC, Lamberty K, Reker D. Management of adult stroke rehabilitation care: A clinical practice guideline. Stroke. 2005;36:e100e43.

5. O'Gara PT, Kushner F, Ascheim D, Casey DJ, Chung M, de Lemos J, Ettinger S, Fang J, Fesmire F, Franklin B, Granger C, Krumholz H, Linderbaum J, Morrow D, Newby L, Ornato J, Ou N, Radford M, Tamis-Holland J, Tommaso C, Tracy C, Woo Y, Zhao D. ACCF/AHA guideline for the management of STelevation myocardial infarction: Executive Summary: A report of the American College of Cardiology Foundation/American Heart Association Task Force on Practice Guidelines. Circulation. 2013;127:52955.

6. Aben I, Verhey F, Strik J, Lousberg R, Lodder J, Honig A. A comparative study into the one year cumulative incidence of depression after stroke and myocardial infarction. I Neurol Neurosurg Psychiatry. 2003;74:581-5.

7. Hackett ML, Pickles K. Part I: Frequency of depression after stroke: A updated systematic review and meta - analysis of observational studies. International Journal of Stroke. 2014;9:1017-25. 8. Meijer A, Conradi HJ, Bos EH, Thombs BD, van Melle JP, de Jonge P. Prognostic association of depression following myocardial infarction with mortality and cardiovascular events: A meta-analysis of 25 years of research. Gen Hosp Psychiatry. 2011;33:203-16.

9. Towfighi A, Ovbiagele B, El Husseini N, Hackett ML, Jorge RE, Kissela BM, Mitchell PH, Skolarus LE, Whooley MA, Williams LS. Poststroke depression: A scientific statement for healthcare professionals from the American Heart Association/American Stroke Association. Stroke. 2017;48:e30-e43.

10. Gehi AK, Ali S, Na B, Whooley MA. Self-reported medication adherence and cardiovascular events in patients with stable coronary heart disease: The heart and soul study. Arch Intern Med. 2007;167:1798-803.

11. Hackett ML, Anderson CS, House A, Xia J. Interventions for treating depression after stroke. Cochrane Libr. 2008.

12. Baumeister $\mathrm{H}$, Hutter $\mathrm{N}$, Bengel J. Psychological and pharmacological interventions for depression in patients with coronary artery disease. Cochrane Libr. 2011.

13. van Dixhoorn J, White A. Relaxation therapy for rehabilitation and prevention in ischaemic heart disease: a systematic review and meta-analysis. Eur J Cardiovasc Prev Rehabil. 2005;12:193-202.

14. Lichtman JH, Bigger JT, Blumenthal JA, Frasure-Smith N, Kaufmann PG, Lespérance F, Mark DB, Sheps DS, Taylor CB, Froelicher ES. Depression and coronary heart disease. Circulation. 2008;118:1768-75.

15. Lindsay $P$, Bayley $M$, Hellings $C$, Hill $M$, Woodbury $E$, Phillips $S$. Canadian best practice recommendations for stroke care (updated 2008). CMAJ. 2008;179:S1-S25.

16. Grover S, Suchendra K, Mehra A, Parkash V, Saini V, Bagga S. Use of electroconvulsive therapy in an elderly after 5 weeks of myocardial infraction with $30 \%$ cardiac output. Ind Psychiatry J. 2015;24:195-7.

17. Bueno VF, Brunoni AR, Boggio PS, Bensenor IM, Fregni F. Mood and cognitive effects of transcranial direct current stimulation in post-stroke depression. Neurocase. 2011;17:318-22. 
18. Jorm AF, Medway J, Christensen H, Korten AE, Jacomb PA, Rodgers B. Public beliefs about the helpfulness of interventions for depression: Effects on actions taken when experiencing anxiety and depression symptoms. Aust N Z J Psychiatry. 2000;34:619-26.

19. Mead GE, Hsieh C-F, Lee R, Kutlubaev M, Claxton A, Hankey GJ, Hackett M. Selective serotonin reuptake inhibitors for stroke recovery. Stroke. 2013;44:844-50.

20. Hesami O, Gharagozli K, Beladimoghadam N, Assarzadegan F, Mansouri B, Sistanizad M. The efficacy of gabapentin in patients with central post-stroke pain. Iran J Pharm Res. 2015;14:95-101.

21. Van Melle JP, De Jonge P, Honig A, Schene AH, Kuyper AM, Crijns HJ, Schins A, Tulner D, Ormel J. Effects of antidepressant treatment following myocardial infarction. The British Journal of Psychiatry. 2007;190:460-6.

22. Carney RM, Blumenthal JA, Freedland KE, Youngblood M, Veith RC, Burg MM, Cornell C, Saab PG, Kaufmann PG, Czajkowski SM. Depression and late mortality after myocardial infarction in the Enhancing Recovery in Coronary Heart Disease (ENRICHD) study. Psychosom Med. 2004;66:466-74.

23. Glassman AH, Bigger JT, Gaffney M. Psychiatric characteristics associated with long-term mortality among 361 patients having an acute coronary syndrome and major depression: Seven-year follow-up of SADHART participants. Arch Gen Psychiatry. 2009;66:1022-9.

24. Joynt KE, O'Connor CM. Lessons from SADHART, ENRICHD, and other trials. Psychosom Med. 2005;67:S63-S6.

25. Gaete JM, Bogousslavsky J. Post-stroke depression. Expert Rev Neurother. 2008;8:75-92.

26. Celano CM, Huffman JC. Depression and cardiac disease: A review. Cardiol Rev. 2011;19:130-

27. House A. Depression after stroke. Br Med J (Clin Res Ed). 1987;294:76-8

28. Lindén T, Blomstrand C, Skoog I. Depressive disorders after 20 months in elderly stroke patients. Stroke. 2007;38:1860-3.

29. Musselman DL, Evans DL, Nemeroff CB. The relationship of depression to cardiovascular disease: epidemiology, biology, and treatment. Arch Gen Psychiatry. 1998;55:580-92.

30. Sundquist K, Chang BP, Parsons F, Dalrymple N, Edmondson D, Sumner JA. Treatment rates for PTSD and depression in recently hospitalized cardiac patients. J Psychosom Res. 2016;86:60-2.

31. Stroup DF, Berlin JA, Morton SC, Olkin I, Williamson GD, Rennie D, Moher D, Becker BJ, Sipe TA, Thacker SB. Meta-analysis of observational studies in epidemiology: A proposal for reporting. J Am Med Assoc. 2000;283:2008-12.

32. Moher D, Liberati A, Tetzlaff J, Altman DG. Preferred reporting items for systematic reviews and meta-analyses: The PRISMA statement. Ann Intern Med. 2009;151:264-9.

33. (WHO) WHO. List of WHO member states and respective world region. 2017 [11/22/2017];

Available from: http://www.who.int/choice/demography/regions/en/.

34. DerSimonian R, Laird N. Meta-analysis in clinical trials. Control Clin Trials. 1986;7:177-88.

35. Egger M, Smith GD, Schneider M, Minder C. Bias in meta-analysis detected by a simple, graphical test. Br Med J. 1997;315:629-34.

36. Paolucci S, Gandolfo C, Provinciali L, Torta R, Toso V, Group tDS. The Italian multicenter observational study on post-stroke depression (DESTRO). J Neurol. 2006;253:556-62.

37. Ayis SA, Ayerbe L, Crichton SL, Rudd AG, Wolfe CDA. The natural history of depression and trajectories of symptoms long term after stroke: The prospective south London stroke register. J Affect Disord. 2016;194:65-71.

38. Townend B, Whyte S, Desborough T, Crimmins D, Markus R, Levi C, Sturm J. Longitudinal prevalence and determinants of early mood disorder post-stroke. J Clin Neurosci. 2007;14:429-34.

39. Townend E, Tinson D, Kwan J, Sharpe M. 'Feeling sad and useless': An investigation into personal acceptance of disability and its association with depression following stroke. Clin Rehabil. 2010;24:554-64.

40. Appelros $\mathrm{P}$, Viitanen $\mathrm{M}$. Prevalence and predictors of depression at one year in a Swedish population-based cohort with first-ever stroke. J Stroke Cerebrovasc Dis. 2004;13:52-7. 
41. Sharpe M, Hawton K, Seagroatt V, Bamford J, House A, Molyneux A, Sandercock P, Warlow C. Depressive disorders in long-term survivors of stroke: Associations with demographic and social factors, functional status, and brain lesion volume. Br J Psychiatry. 1994;164:380-6.

42. Skånér $Y$, Nilsson GH, Sundquist K, Hassler E, Krakau I. Self-rated health, symptoms of depression and general symptoms at 3 and 12 months after a first-ever stroke: A municipality-based study in Sweden. BMC Fam Pract. 2007;8.

43. Tene O, Shenhar-Tsarfaty S, Korczyn AD, Kliper E, Hallevi H, Shopin L, Auriel E, Mike A, Bornstein NM, Assayag EB. Depressive symptoms following stroke and transient ischemic attack: Is it time for a more intensive treatment approach? results from the TABASCO cohort study. J Clin Psychiatry. 2016;77:673-80.

44. Celano CM, Beale EE, Beach SR, Belcher AM, Suarez L, Motiwala SR, Gandhi PU, Gaggin H, Januzzi Jr JL, Healy BC. Associations between pychological constructs and cardiac biomarkers after acute coronary syndrome. Psychosom Med. 2017;79:318-26.

45. Frasure-Smith N, Lespérance F, Talajic M. Depression following myocardial infarction: impact on 6-month survival. J Am Med Assoc. 1993;270:1819-25.

46. Grace SL, Abbey SE, Pinto R, Shnek ZM, Irvine J, Stewart DE. Longitudinal course of depressive symptomatology after a cardiac event: Effects of gender and cardiac rehabilitation. Psychosom Med. 2005;67:52-8.

47. Grace SL, Leung YW, Stewart DE. A prospective examination of antidepressant use and its correlates in patients with acute coronary syndrome. Psychosomatics. 2008;49:199-207.

48. Huffman JC, Smith FA, Blais MA, Beiser ME, Januzzi JL, Fricchione GL. Recognition and treatment of depression and anxiety in patients with acute myocardial infarction. Am J Cardiol. 2006;98:319-24.

49. Kroemeke A. Depressive symptom trajectories over a 6-year period following myocardial infarction: Predictive function of cognitive appraisal and coping. J Behav Med. 2016;39:181-91.

50. Kuhlmann SL, Tschorn M, Arolt V, Beer K, Brandt J, Grosse L, Haverkamp W, Müller-Nordhorn J, Rieckmann N, Waltenberger J. Serum brain-derived neurotrophic factor and stability of depressive symptoms in coronary heart disease patients: A prospective study. Psychoneuroendocrinology. 2017;77:196-202.

51. Parakh K, Thombs BD, Fauerbach JA, Bush DE, Ziegelstein RC. Effect of depression on late (8 years) mortality after myocardial infarction. Am J Cardiol. 2008;101:602-6.

52. Rothenbacher D, Jaensch A, Mons U, Hahmann H, Becker T, Koenig W, Brenner H. Prognostic value of one-year course of symptoms of anxiety and depression in patients with coronary heart disease: Role of physical activity and unmet medical need. Eur J Prev Cardiol. 2015;22:1129-38.

53. Schlyter M, Östman M, Engström G, André-Petersson L, Tydén P, Leosdottir M. Personality factors and depression as predictors of hospital-based health care utilization following acute myocardial infarction. Eur J Cardiovasc Nurs. 2017;16:318-25.

54. Volz M, Möbus J, Letsch C, Werheid K. The influence of early depressive symptoms, social support and decreasing self-efficacy on depression 6 months post-stroke. Journal of Affective Disorders. 2016;206:252-5.

55. Carod-Artal J, Egido JA, González JL, De Seijas EV. Quality of life among stroke survivors evaluated 1 year after stroke: Experience of a stroke unit. Stroke. 2000;31:2995-3000.

56. Ebrahim S, Barer D, Nouri F. Affective illness after stroke. Br J Psychiatry. 1987;151:52-6.

57. El Husseini N, Goldstein LB, Peterson ED, Zhao X, Pan W, Olson DM, Zimmer LO, Williams JW, Bushnell C, Laskowitz DT. Depression and antidepressant use after stroke and transient ischemic attack. Stroke. 2012;43:1609-16.

58. Kauhanen M, Korpelainen J, Hiltunen $P$, Brusin E, Mononen $H$, Määttä R, Nieminen $P$, Sotaniemi K, Myllylä V. Poststroke depression correlates with cognitive impairment and neurological deficits. Stroke. 1999;30:1875-80.

59. Kotila M, Numminen $\mathrm{H}$, Waltimo O, Kaste M. Post - stroke depression and functional recovery in a population - based stroke register. The Finnstroke study. Eur J Neurol. 1999;6:309-12. 
60. Paolucci S, Di Vita A, Massicci R, Traballesi M, Bureca I, Matano A, losa M, Guariglia C. Impact of participation on rehabilitation results: A multivariate study. Eur J Phys Rehabil Med. 2012;48:455-

66.

61. Pohjasvaara T, Leppävuori A, Siira I, Vataja R, Kaste M, Erkinjuntti T. Frequency and clinical determinants of poststroke depression. Stroke. 1998;29:2311-7.

62. Cassidy EM, O'Connor R, O'Keane V. Prevalence of post-stroke depression in an Irish sample and its relationship with disability and outcome following inpatient rehabilitation. Disabil Rehabil. [Article]. 2004;26:71-7.

63. De Ryck A, Fransen E, Brouns R, Geurden M, Peij D, Mariën P, De Deyn PP, Engelborghs S. Psychosocial problems associated with depression at 18 months poststroke. Int J Geriatr Psychiatry. 2014;29:144-52.

64. Desmaele S, Putman K, De Wit L, Dejaeger E, Gantenbein AR, Schupp W, Steurbaut S, Dupont AG, De Paepe K. A comparative study of medication use after stroke in four countries. Clin Neurol Neurosurg. [Article]. 2016;148:96-104.

65. Jürgensen F, Meins W, Meier-Baumgartner HP. Depression after stroke: Prevalence, functional outcome and recovery six months after discharge from a geriatric rehabilitation center. Z Gerontol Geriatr. 1999;32:251.

66. Naess $\mathrm{H}$, Lunde L, Brogger J. The triad of pain, fatigue and depression in ischemic stroke patients: The Bergen Stroke Study. Cerebrovasc Dis. 2012;33:461-5.

67. Nys G, Van Zandvoort M, Van der Worp H, De Haan E, De Kort P, Kappelle L. Early depressive symptoms after stroke: Neuropsychological correlates and lesion characteristics. J Neurol Sci. 2005;228:27-33.

68. van de Weg F, Kuik D, Lankhorst G. Post-stroke depression and functional outcome: A cohort study investigating the influence of depression on functional recovery from stroke. Clin Rehabil. 1999;13:268-72.

69. Verdelho A, Henon H, Lebert F, Pasquier F, Leys D. Depressive symptoms after stroke and relationship with dementia: A three-year follow-up study. Neurology. 2004;62:905-11.

70. Paul SL, Dewey HM, Sturm JW, Macdonell RA, Thrift AG. Prevalence of depression and use of antidepressant medication at 5-years poststroke in the North East Melbourne Stroke Incidence Study. Stroke. 2006;37:2854-5.

71. Loong CK, Kenneth NKC, Paulin ST. Post-stroke depression: Outcome following rehabilitation. Aust N Z J Psychiatry. 1995;29:609-14.

72. Saxena S, Ng T, Yong D, Fong N, Koh G. Subthreshold depression and cognitive impairment but not demented in stroke patients during their rehabilitation. Acta Neurol Scand. 2008;117:133-40.

73. Shi YZ, Xiang YT, Wu SL, Zhang N, Zhou J, Bai Y, Wang S, Wang YL, Zhao XQ, Ungvari GS, Chiu HF, Wang YJ, Wang CX. The relationship between frontal lobe lesions, course of post-stroke depression, and 1-year prognosis in patients with first-ever ischemic stroke. PLoS One. [Research Support, Non-U.S. Gov't]. 2014;9:e100456.

74. Hayee M, Akhtar N, Haque A, Rabbani M. Depression after stroke-analysis of 297 stroke patients. Bangladesh Med Res Counc Bull. 2001;27:96-102.

75. Chausson N, Olindo S, Cabre P, Saint-Vil M, Smadja D. Five-Year Outcome of a Stroke Cohort in Martinique, French West Indies: Etude Réalisée en Martinique et Centrée sur l'Incidence des Accidents vasculaires cérebraux, Part 2. Stroke. 2010;41:594-9.

76. Bacher Y, Korner-Bitensky N, Mayo N, Becker R. A longitudinal study of depression among stroke patients participating in a rehabilitation program. Can J Rehabil. 1990;4:27-37.

77. Herrmann N, Black S, Lawrence J, Szekely C, Szalai J. The Sunnybrook stroke study: A prospective study of depressive symptoms and functional outcome. Stroke. 1998;29:618-24.

78. Coyle MK. Depressive symptoms after a myocardial infarction and self-care. Arch Psychiatr Nurs. 2012;26:127-34. 
79. Lauzon C, Beck CA, Huynh T, Dion D, Racine N, Carignan S, Diodati JG, Charbonneau F, Dupuis $\mathrm{R}$, Pilote L. Depression and prognosis following hospital admission because of acute myocardial infarction. CMAJ. 2003;168:547-52.

80. Mallik S, Spertus JA, Reid KJ, Krumholz HM, Rumsfeld JS, Weintraub WS, Agarwal P, Santra M, Bidyasar S, Lichtman JH. Depressive symptoms after acute myocardial infarction: Evidence for highest rates in younger women. Arch Intern Med. 2006;166:876-83.

81. Smolderen KG, Buchanan DM, Gosch K, Whooley MA, Chan PS, Vaccarino V, Parashar S, Shah AJ, Ho PM, Spertus JA. Depression treatment and 1-year mortality following acute myocardial infarction: Insights from the TRIUMPH registry. Circulation. 2017:CIRCULATIONAHA. 116.025140.

82. Travella JI, Forrester AW, Schultz SK, Robinson RG. Depression following myocardial infarction: a one year longitudinal study. Int J Psychiatry Med. 1994;24:357-69.

83. Larsen KK, Christensen B, Søndergaard J, Vestergaard M. Depressive symptoms and risk of new cardiovascular events or death in patients with myocardial infarction: A population-based longitudinal study examining health behaviors and health care interventions. PLoS One. 2013;8:e74393.

84. Spijkerman T, de Jonge P, van den Brink RH, Jansen JH, May JF, Crijns HJ, Ormel J. Depression following myocardial infarction: First-ever versus ongoing and recurrent episodes. Gen Hosp Psychiatry. 2005;27:411-7.

85. Huffman JC, Mastromauro CA, Beach SR, Celano CM, DuBois CM, Healy BC, Suarez L, Rollman $\mathrm{BL}$, Januzzi JL. Collaborative care for depression and anxiety disorders in patients with recent cardiac events: The Management of Sadness and Anxiety in Cardiology (MOSAIC) randomized clinical trial. JAMA Intern Med. 2014;174:927-35.

86. Jorge RE, Robinson RG, Arndt S, Starkstein S. Mortality and poststroke depression: A placebocontrolled trial of antidepressants. American Journal of Psychiatry. 2003;160:1823-9.

87. Eriksson M, Asplund K, Glader E-L, Norrving B, Stegmayr B, Terént A, Åsberg KH, Wester P-O. Self-reported depression and use of antidepressants after stroke: A national survey. Stroke. 2004;35:936-41.

88. Gustafson Y, Nilsson I, Mattsson M, Åström M, Bucht G. Epidemiology and treatment of poststroke depression. Drugs Aging. 1995;7:298-309.

89. Corrigan P. How stigma interferes with mental health care. Am Psychol. 2004;59:614-25.

90. Simon GE, VonKorff M. Recognition, management, and outcomes of depression in primary care. Arch Fam Med. 1995;4:99-105.

91. Mojtabai R, Olfson M, Mechanic D. Perceived need and help-seeking in adults with mood, anxiety, or substance use disorders. Arch Gen Psychiatry. 2002;59:77-84. 